\title{
Trends in Inpatient Urological Surgery Practice Patterns
}

\author{
Scott R. Hawken, MS, Lindsey A. Herrel, MD MS, Chandy Ellimoottil, MD MS, Zaojun Ye, \\ MS, J. Quentin Clemens, MD MS, and David C. Miller, MD, MPH \\ Dow Division of Health Services Research, Department of Urology, University of Michigan, Ann \\ Arbor, MI
}

\section{Abstract}

Introduction-As the nation's population ages and the number of practicing urologists per capita declines, characterization of practice patterns is essential to understand the current state of the urological workforce and anticipate future needs. Accordingly, we examined trends in adult inpatient urological surgery practice patterns over a five-year period.

Methods-We used the Nationwide Inpatient Sample (NIS) data from 2005 through 2009 to identify both surgeons and urological surgeries. We classified the latter into 1 of 7 clinical domains (Endourology \& Stone Disease, Incontinence, Urogenital Reconstruction, Urologic Oncology, Benign Prostate, Renal Transplant, and Other Urological Procedures). For each urological surgeon, three parameters were determined for each year: 1) Case-diversity (the number of distinct urological clinical domains in which they performed $\geq 2$ procedures/year); 2) Subspecialty (the predominant clinical domain of cases that each surgeon performed); and 3) Subspecialty-focus (the proportion of a surgeon's total urological cases/year that belonged to their assigned clinical domain). We examined trends in these metrics over a five-year period, and compared results between urban and rural practice settings.

Results-We analyzed data for 2,237 individual surgeons performing 144,138 inpatient surgeries. Over time, urologist's practice patterns evolved toward lower case-diversity $(\mathrm{p}<0.001)$ and greater subspecialty-focus $(\mathrm{p}<0.001)$. These trends were more pronounced for surgeons practicing in urban versus rural practice settings (p-values $<0.05$ ).

Conclusions-At a national level, urologists' inpatient surgical practice patterns are narrowing, with less case-diversity and higher subspecialty-focus. These trends are even more prominent among urologists in urban, compared with rural, practice settings.

\section{Keywords}

Workforce; Urology; Physician Practice Patterns; Urological Surgical Procedures

Corresponding Author: David C. Miller, MD, MPH, Associate Professor, Department of Urology, University of Michigan, NCRC Building 16, Room 108E, 2800 Plymouth Road, Ann Arbor, MI 48109-2800, Phone: (734) 936-0054, Fax: (734) 232-2400, dcmiller@med.umich.edu.

Publisher's Disclaimer: This is a PDF file of an unedited manuscript that has been accepted for publication. As a service to our customers we are providing this early version of the manuscript. The manuscript will undergo copyediting, typesetting, and review of the resulting proof before it is published in its final citable form. Please note that during the production process errors may be discovered which could affect the content, and all legal disclaimers that apply to the journal pertain. 


\section{Introduction}

The United States' population is aging, creating greater demand for urological and other medical services. ${ }^{1,2}$ Concurrently, the overall number of urologists per capita is declining. ${ }^{3-5}$ Moreover, the surgical practice patterns for many urologists are being influenced and modified by several factors, including the movement towards large group practices, preferences for urban practice settings, and increasing clinical sub-specialization. 3,6,7 Collectively, these developments have raised some concerns about imperiled access for patients in need of urological specialty care, particularly in rural areas. ${ }^{3}$

One way to explore these concerns is to examine longitudinal trends in urologists' inpatient surgical practice patterns, including differences according to urban versus rural practice settings. By evaluating changes in the diversity of inpatient procedures performed, one can gain insight to whether or not the breadth of surgical care provided by most urologists is expanding or contracting. In addition to case diversity, assessment of inpatient practice patterns provides insight around trends in subspecialty focus - i.e., the degree to which urologists' caseloads are comprised of procedures from their predominant clinical subspecialty. Taken together, such data will inform the degree to which contemporary urologists are maintaining (or not) a broad surgical practice.

In this context, we used the Nationwide Inpatient Sample to investigate trends in urologists' inpatient surgical practice patterns. Ultimately, a better understanding of current trends will aid efforts to anticipate workforce needs in the foreseeable future.

\section{Methods \\ Data Source}

We conducted a retrospective cohort study of surgeons performing inpatient urological procedures in hospitals included in the Nationwide Inpatient Sample (NIS) from 2005 through 2009. The NIS contains approximately 8 million standard hospital discharge abstracts from 44 states, approximating a 20 percent stratified sample of all community hospitals in the US. ${ }^{8}$ The discharge abstracts include patient demographics, ICD-9-CM diagnosis and procedures codes, encrypted physician and surgeon identifiers, hospital location (urban or rural as defined by Core Based Statistical Area codes), and admission and discharge information.

\section{Identification of Surgical Procedures}

We identified adult ( $\geq 18$ years old) inpatient surgical procedures by the presence of a principal ICD-9-CM procedure code meeting NIS criteria for a major procedure performed in an operating room. ${ }^{9}$ Next, we used Clinical Classification Software (CCS) ${ }^{10}$ based on ICD-9-CM codes to identify all major inpatient urological procedures (Appendix). ${ }^{9}$ At the time of the study, the ICD-10-CM system for inpatient hospital coding had not yet been adopted in the U.S. 


\section{Identification of Urological Surgeons}

For each year of data, we used an encrypted surgeon identifier present in the NIS to identify unique surgeons, and we excluded states with inconsistent reporting of this variable. ${ }^{11,12}$ To classify surgeons as urologists, we required that they performed $\geq 10$ major inpatient urological procedures in a given year, ${ }^{13}$ and that the majority of their inpatient surgeries were major urological procedures. Surgeons meeting both criteria were defined as urologists. We classified each urologist as practicing in an urban or rural setting based on the location of the hospital where they performed a majority of their inpatient urologic procedures.

\section{Classifying Urological Procedures}

Our goal for this analysis was to examine urologists' inpatient surgical practice patterns, including the overall breadth of procedures performed, and the degree of focus on specific clinical domains of urological procedures. To do this, we first assigned each urological ICD-9-CM procedure code into one of seven mutually exclusive and clinically relevant domains, including: Endourology \& Stone Disease, Incontinence, Urogenital Reconstruction, Urologic Oncology, Benign Prostate, Renal Transplant, and Other Urological Procedures (primarily lysis of adhesions and suprapubic cystostomy) (Appendix). ${ }^{14}$ Throughout the manuscript, we refer to each of these categories as a urological "clinical domain".

\section{Case-Diversity}

Our first measure of interest was the case-diversity of each urologist, defined for this analysis as the total number of distinct clinical domains where each urologist performed at least 2 major urological procedures. This measure reflects the breadth of conditions and procedures comprising a urologist's inpatient surgical practice. For analytic purposes, we defined urologists as having "high case-diversity" if they performed inpatient procedures from more than 4 distinct clinical domains, and "low case-diversity" if they performed major inpatient urological procedures from 4 or fewer clinical domains.

\section{Subspecialty}

Our second measure of interest was the predominant clinical domain of procedures for each urologist. To calculate this measure, we first determined the clinical domain (e.g., Endourology \& Stone disease, Incontinence, etc.) that comprised the largest absolute number of the surgeon's total inpatient case mix per year. We defined the urologist as belonging to that particular "subspecialty". If there were an equal number of cases in several clinical domains in a given year, we assigned that urologist to their subspecialty designation in other years. We excluded urologists with a tie between subspecialties and only one year of data.

\section{Subspecialty-Focus}

Next, we calculated the proportion of each surgeon's total urological cases per year comprised of procedures in their assigned subspecialty, i.e., the proportion of all of the inpatient surgeries in a given year from their clinical domain. ${ }^{15}$ We defined this percentage 
as the urologist's "subspecialty-focus," because it reflects concentration on a single group of urological conditions. For urologists in general practice, as well as subspecialists, this measure allows us to quantify the surgeon's degree of focus on a particular clinical domain.

\section{Statistical Analyses}

In our first analytic step, we fit a multivariable logistic regression model to examine changes in case-diversity over time, as well as the differences in case-diversity between urologists practicing in urban versus rural settings. Our outcome, low (versus high) case-diversity was treated as a dichotomous variable. Practice setting (urban versus rural) was also treated as a dichotomous variable, and year as a continuous variable. We also tested for an interaction between the effects of year and urban (versus rural) practice on case-diversity. To account for repeated measures at the surgeon level, we used generalized estimating equations. To evaluate the impact of assigned subspecialty on case-diversity, we performed a sensitivity analysis that included surgeon subspecialty as a categorical variable in the model.

In our second analytic step, we examined the change in subspecialty-focus over time by fitting a multivariable Poisson regression model. The outcome, subspecialty-focus, was treated as a discrete proportion. Again, practice setting (urban versus rural) was treated as a dichotomous variable and year as a continuous variable. To test whether subspecialty-focus changed at a different rate in urban vs. rural settings, we tested for an interaction between the effects of year and practice setting. Generalized estimating equations were again used to account for repeated measures at the surgeon level.

Finally, we examined whether the trends in subspecialty-focus were different across clinical domains. We used the model described previously, but also included the assigned subspecialty as a categorical variable. Furthermore, to examine whether subspecialty-focus changed at a different rate across subspecialties, we again tested for an interaction between the effects of year and assigned clinical domain. We then plotted the predicted trends in subspecialty-focus for those groups that contained at least $10 \%$ of the total cohort of urologists, including Endourology \& Stone Disease, Urologic Oncology, and Benign Prostate.

All statistical testing was performed at the 5\% significance level using SAS v9.3 (SAS Institute Inc., Cary, NC, USA). This study was deemed exempt from review by the University of Michigan institutional review board.

\section{Results}

We identified 2,317 individual urologists that performed major inpatient procedures over the 5 -year period. Among this group, 80 surgeons (3\%) were either missing data on practice location or could not be assigned a subspecialty, and were therefore excluded from the analysis. Because individual surgeons were tracked over multiple years, we ultimately examined practice patterns for 3,218 urological surgeon-years. Table 1 displays the total number of surgeons identified each year. These surgeons practiced in 19 states, representing all four hospital census regions (Northeast, Midwest, South, and West). The median percentage of total cases/year that were urological procedures was 94.5\% (IQR: 81.5-100\%), 
and the median number of urological procedures/year was 31 (IQR: 17-55). Overall, 8.3\% of these surgeons practiced in a rural location.

The proportion of all surgeons with low case-diversity (i.e., performed cases in $\$ 4$ clinical domains) is presented by year and practice setting in Figure 1. In multivariable analyses, the adjusted likelihood of a surgeon having low case-diversity increased over time (OR 1.2, 95\% CI: 1.1-1.2, $\mathrm{p}<0.001)$. Moreover, urologists practicing in an urban location were more likely to have low case-diversity (OR 1.8, 95\% CI: 1.1-3.0, $\mathrm{p}=0.03$ ). There was not a significant interaction between year and practice setting $(\mathrm{p}=0.2)$. Our results did not change in a sensitivity analysis that also adjusted for each surgeon's assigned clinical subspecialty, suggesting that these trends are consistent regardless of urologists' predominant clinical domain.

Table 1 presents the distribution of surgeons according to predominant clinical domain for each year. For the entire cohort, the degree of subspecialty-focus increased by $3.9 \%$ annually (95\% CI: 2.4-5.5\%, p<0.001). In other words, each year urologists have performed 3.9\% more cases in their most predominant clinical domain, relative to their entire inpatient surgery case load. Furthermore, urological surgeons practicing in urban settings had 17\% greater subspecialty-focus than those practicing in rural settings (95\% CI: 8.2-28\%, $\mathrm{p}<0.001$ ), indicating that urban urologists' practice patterns are more concentrated on their predominant clinical domain. There was no significant interaction between year and practice setting $(\mathrm{p}=0.9)$. Figure 2 presents the adjusted trends in subspecialty-focus stratified by practice setting.

When we examined the trends in subspecialty-focus according to specific clinical domains, we found that surgeons assigned to Urologic Oncology had significantly greater subspecialty-focus: $16 \%$ and $29 \%$ greater than for urologists assigned to Endourology \& Stone Disease (95\% CI: 9-22\%, p<0.001) and Benign Prostate (95\% CI: 23-46\%, p<0.001), respectively. However, there was no significant difference in the rate of change in subspecialty-focus across these clinical domains ( $p>0.05$ for each comparison). Figure 3 displays the adjusted trends in subspecialty-focus for surgeons assigned to Endourology \& Stone Disease, Urologic Oncology, and Benign Prostate.

\section{Discussion}

We examined trends in adult inpatient urological surgery practice patterns, as measured by breadth of surgical practice (case-diversity) and concentration in a clinical domain (subspecialty-focus). Over time, we found urologists tended to operate on a narrower range of urological conditions (lower case-diversity), with increasing concentration on cases from their most predominant clinical domain (higher subspecialty-focus). These trends were more prominent for surgeons in urban versus rural practice settings. Collectively, these findings indicate that urologists are becoming more sub-specialized over time, at least with respect to their portfolio of inpatient surgeries.

Our findings are consistent with published literature examining the urological workforce and surgical practice patterns in general. Previous studies have demonstrated that urologists are 
increasingly joining group practices; $; 3$ in fact, the AUA Annual Census reports that only $12.3 \%$ of urologists remain in solo practice. ${ }^{16}$ Within group practices, urologists may focus on certain conditions and/or procedures in order to gain greater experience and expertise. ${ }^{17}$ This focus may be more pronounced in larger groups, as a greater number of referrals could potentially be directed towards specific providers that limit their practices to a particular area of expertise. In addition, surgeons' practice patterns may be affected by the number of other specialists serving a patient population or geographic region. ${ }^{15}$ Therefore, it is reasonable to expect that in urban areas, where there are often more urologists per capita, ${ }^{6}$ factors such as competition among providers may encourage surgical sub-specialization. Lastly, as seen in other surgical fields, it is possible that more urologists are pursuing fellowship training, leading to greater sub-specialization. ${ }^{18}$ Overall, it is likely that a combination of surgical demand and personal preferences among urologists are driving this trend in subspecialization.

Our study has several limitations. First, the study period only extends through the year 2009. This is because 2009 was the last year the database reported a surgeon identifier. Still there is no clear reason to expect that these trends would change substantively in more recent years. Second, it is possible that we did not identify every urologist in the NIS, and/or classified some non-urological surgeons as urologists. However, our selection criteria were similar to other published methods, ${ }^{13}$ and resulted in a cohort with a median of $95 \%$ of their total inpatient surgical cases in urology. Third, the use of administrative data precludes us from measuring certain surgeon characteristics that may influence case mix, such as age, fellowship training, and practice structure (e.g., practice size and academic versus private practice). Fourth, due to non-uniform reporting of surgeon identifiers in the NIS, certain states could not be included in our analysis. However, our study did include surgeons from all major geographic regions of the country. Fifth, we only examined inpatient surgical cases, and were unable to capture outpatient procedures. Nevertheless, this all-payer dataset, including hospitals from across the country, still provides a useful lens into inpatient surgical practice patterns.

These limitations notwithstanding, our findings have implications for patients, urologists, and educators. For patients, finding a provider with sub-specialized urological expertise may become easier over time, particularly in urban areas. This could be an important step in meeting the growing demand for surgeons that meet specific volume thresholds for complex inpatient procedures. ${ }^{19}$ However, patients in rural areas could potentially have difficultly accessing care if there are fewer and fewer providers with broad surgical expertise.

For urologists, as more surgeons focus on treating only patients with specific conditions, there may be pressure to further sub-specialize, particularly in urban settings. This could have implications for employment opportunities, particularly for new graduates that choose to forgo fellowship training. Conversely, from an educational perspective, it is possible that fellowship training will be increasingly in-demand, as trainees strive to become more marketable by filling a sub-specialized role in a practice. ${ }^{20,21}$

Moving forward, it will be important to examine the impact of growing sub-specialization on the quality, utilization, and cost of care provided to patients with urological diseases and 
disorders. Likewise, it will be critical to understand whether and to what extent subspecialization and narrowing practice patterns impact access to care for certain populations, including those in already-underserved rural settings. Ultimately, such information could have important implications for the structure and goals of urological training programs.

\section{Acknowledgments}

\section{Funding/Support:}

This work was supported by funding from the National Cancer Institute (1-RO1-CA-174768 to DCM) and the National Center for Advancing Translational Sciences (2UL1TR000433-06 to SRH).

\section{Appendix}




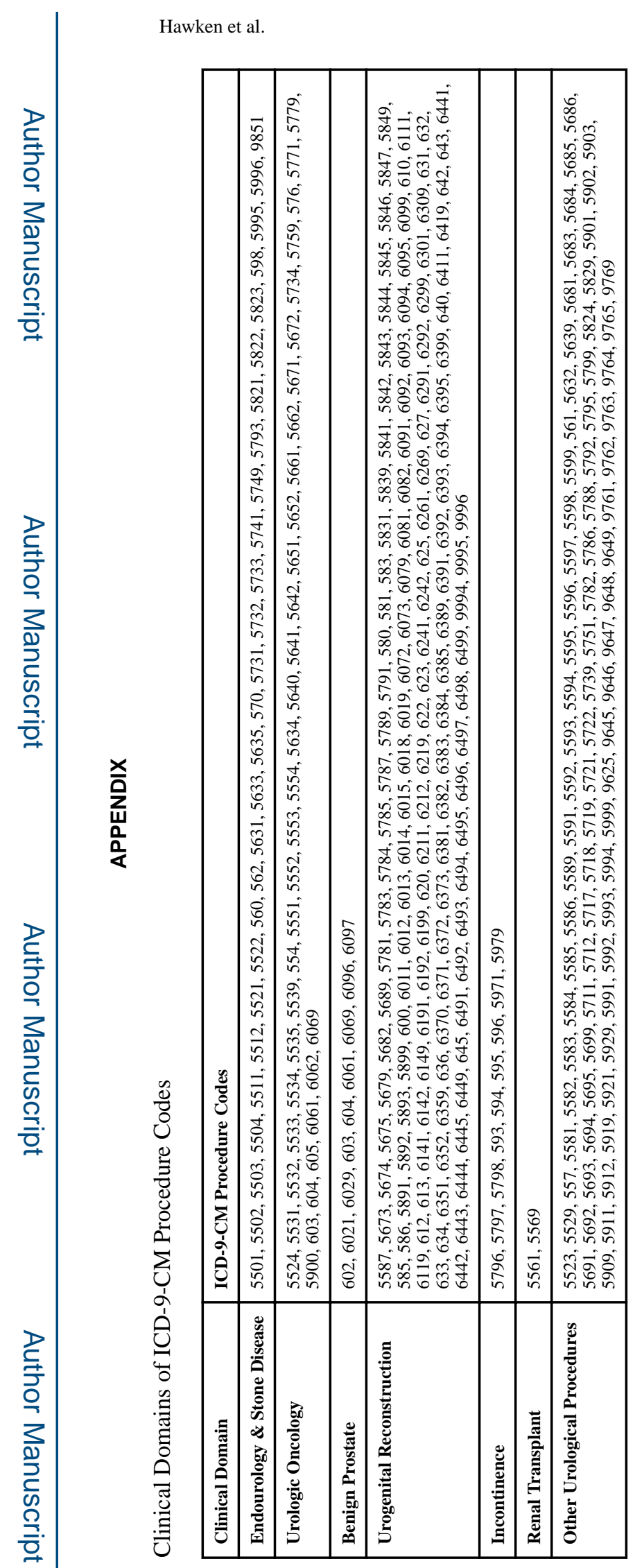

Page 8 


\title{
Key of Definitions for Abbreviations
}

\author{
NIS Nationwide Inpatient Sample \\ ICD-9-CM International Classification of Disease, Ninth Revision, Clinical \\ Modification
}

\section{References}

1. Colby, S.; Ortman, J. Current Population Reports; P25-1143. US Census Bureau; Washington DC: 2014. Projections of the Size and Composition of the U.S. Population: 2014 to 2060. Available at: https://www.census.gov/content/dam/Census/library/publications/2015/demo/p25-1143pdf. [July 8, 2015]

2. Bernstein, AB.; Hing, E.; Moss, AJ., et al. Health care in America: Trends in utilization. National Center for Health Statistics; Hyattsville, Maryland: 2003. Available at: http://www.cdc.gov/nchs/ data/misc/healthcare.pdf. [July 8, 2015]

3. Pruthi RS, Neuwahl S, Nielsen ME, et al. Recent Trends in the Urology Workforce in the United States. Urology. 2013; 82:987-994. [PubMed: 24055244]

4. Gonzalez CM, McKenna P. Challenges facing academic urology training programs: An impending crisis. Urology. 2013; 81:475-479. [PubMed: 23452802]

5. Neuwahl S, Thompson K, Fraher E, et al. HPRI data tracks. Urology Workforce trends. Bull Am Coll Surg. 2012; 97:46-9.

6. Odisho AY, Fradet V, Cooperberg MR, et al. Geographic Distribution of Urologists Throughout the United States Using a County Level Approach. J. Urol. 2009; 181:760-766. [PubMed: 19091334]

7. Sigman M. Is it about business, education, or patient care? Fertil. Steril. 2014; 101:1512-1513. [PubMed: 24739455]

8. Introduction to the HCUP Nationwide Inpatient Sample (NIS). Agency for Healthcare Research and Quality Healthcare Cost and Utilization Project; Rockville, Maryland: 2013. Available at: https:// www.hcup-us.ahrq.gov/db/nation/nis/NIS_Introduction_2011.pdf. [Nov 1, 2014]

9. Gadzinski AJ, Dimick JB, Ye Z, et al. Utilization and outcomes of inpatient surgical care at critical access hospitals in the United States. JAMA Surg. 2013; 148:589-96. [PubMed: 23636896]

10. Clinical Classifications Software for ICD-9-CM. Healthc. Agency for Healthcare Research and Quality Healthcare Cost and Utilization Project; Rockville, Maryland: 2014. Available at: www.hcup-us.ahrq.gov/toolssoftware/ccs/ccs.jsp. [Nov 1, 2014]

11. HCUP NIS Database Documentation. Agency for Healthcare Research and Quality Healthcare Cost and Utilization Project; Rockville, Maryland: 2014. Available at: www.hcup us.ahrq.gov/db/ nation/nis/nisdbdocumentation.jsp. [Nov 1, 2014]

12. Hollingsworth JM, Hollenbeck BK, Englesbe MJ, et al. Operative Mortality After Renal Transplantation-Does Surgeon Type Matter? J. Urol. 2007; 177:2255-2259. [PubMed: 17509333]

13. Hollingsworth JM, Birkmeyer JD, Ye Z, et al. Specialty-Specific Trends in the Prevalence and Distribution of Outpatient Surgery: Implications for Payment and Delivery System Reforms. Surg. Innov. 2014; 21:560-565. [PubMed: 24608183]

14. Gadzinski AJ, Dimick JB, Ye Z, et al. Inpatient urological surgery at critical access hospitals in the United States. J. Urol. 2013; 189:1475-1480. [PubMed: 23041344]

15. Decker MR, Dodgion CM, Kwok AC, et al. Specialization and the current practices of general surgeons. J. Am. Coll. Surg. 2014; 218:8-15. [PubMed: 24210145]

16. American Urological Association. The State of Urology Workforce and Practice in the United States 2014. Linthicum, Maryland: 2015. Available at: https://www.auanet.org/common/pdf/ research/census/State-Urology-Workforce-Practice-US.pdf. [July 8, 2015]

17. Dagrosa LM, Pais VM, Assimos D, et al. Stone Specialist, Cancer Surgeon, or Both? Optimizing the Endourology Fellowship Training Model. J. Endourol. 2015; 29:75-77. [PubMed: 25000513]

18. Stitzenberg KB, Sheldon GF. Progressive specialization within general surgery: Adding to the complexity of workforce planning. J. Am. Coll. Surg. 2005; 201:925-932. [PubMed: 16310697] 
19. Birkmeyer, J.; Pronovost, P. [August 10, 2015] Why We Need Minimum Volume Standards. US. News World Rep. 2015. Available at: http://health.usnews.com/health-news/blogs/second-opinion/ 2015/06/23/why we-need-minimum-volume-standards.

20. Nayak JG, Drachenberg DE, Mau E, et al. The impact of fellowship training on pathological outcomes following radical prostatectomy: a population based analysis. BMC Urol. 2014; 14:82. [PubMed: 25339410]

21. Touma NJ, Siemens DR. Attitudes and experiences of residents in pursuit of postgraduate fellowships : A national survey of Canadian trainees. Can. Urol. Assoc. J. 2014; 8:437-441. [PubMed: 25553159] 


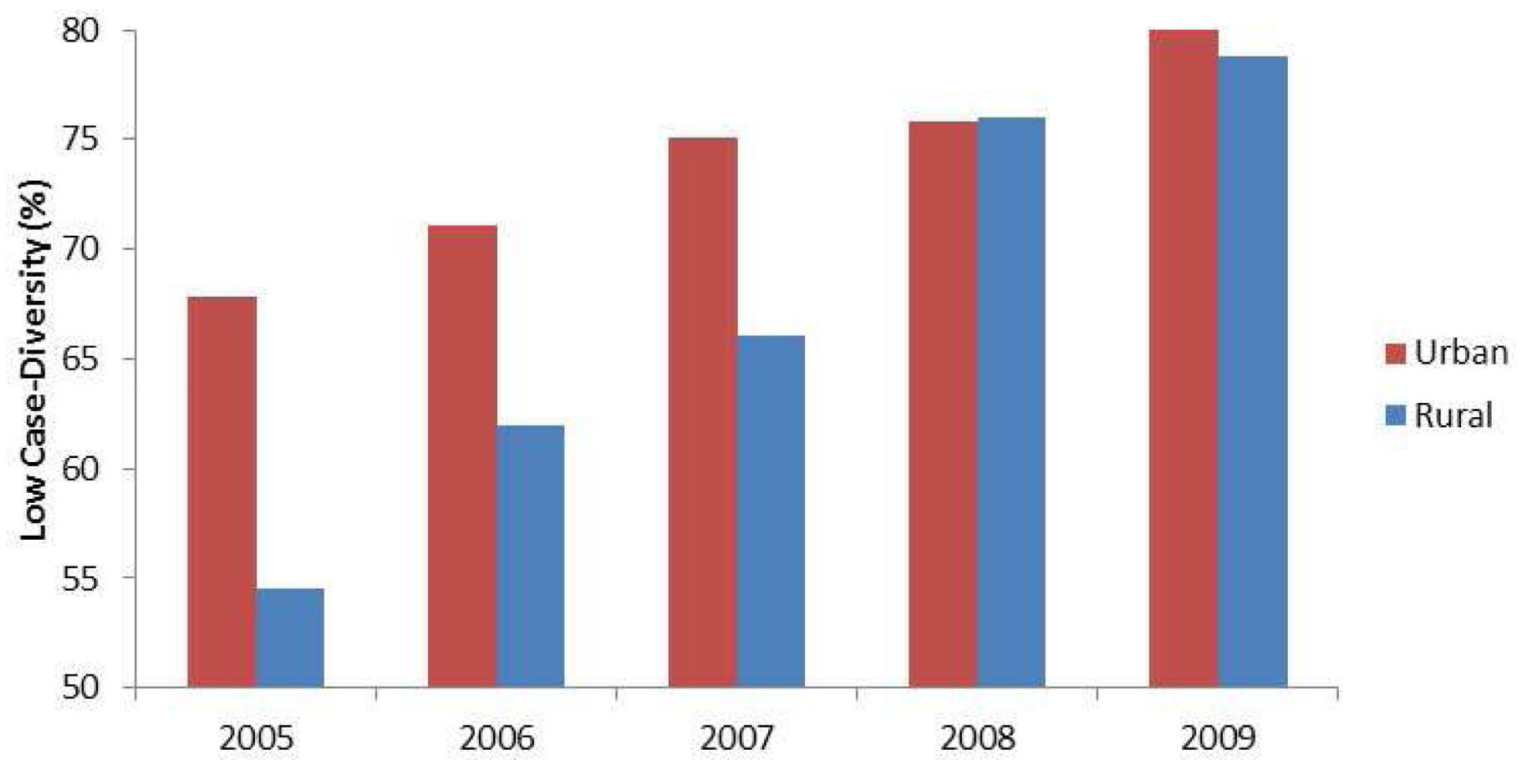

Figure 1. Longitudinal trends in case-diversity according to practice setting Annual proportion of urologists with low case-diversity. Both year and urban practice setting were associated with a lower case-diversity ( $\mathrm{p}<0.001, \mathrm{p}=0.03$ respectively). 


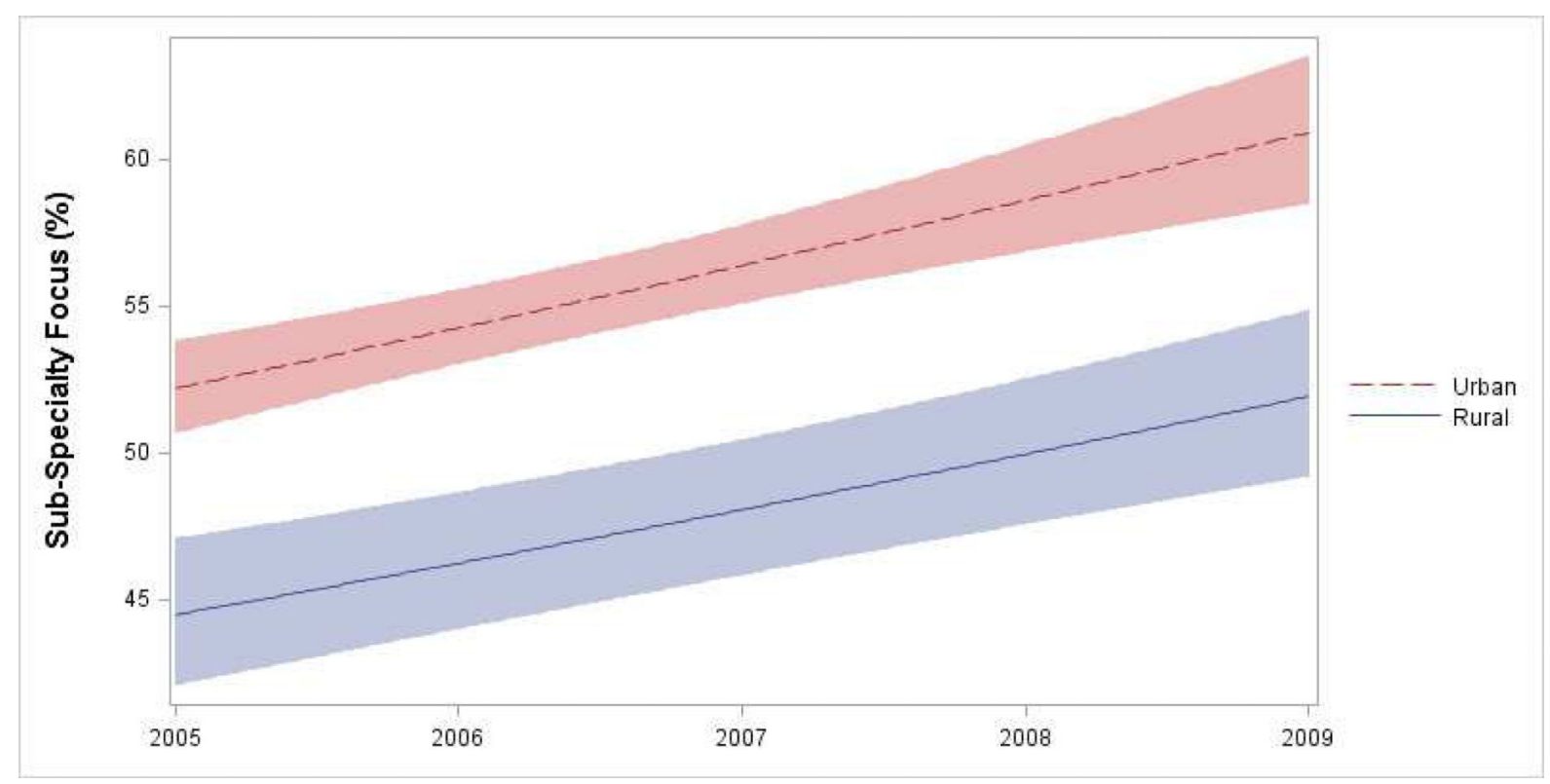

Figure 2. Longitudinal trends in subspecialty-focus according to practice setting Trends in subspecialty-focus by practice setting, with $95 \%$ confidence intervals (shaded areas). Year and urban practice setting were associated with greater subspecialty-focus $(\mathrm{p}<0.001$ for each), but the rate of change in subspecialty-focus was statistically similar for urologists practicing urban and rural settings $(\mathrm{p}=0.9)$. 


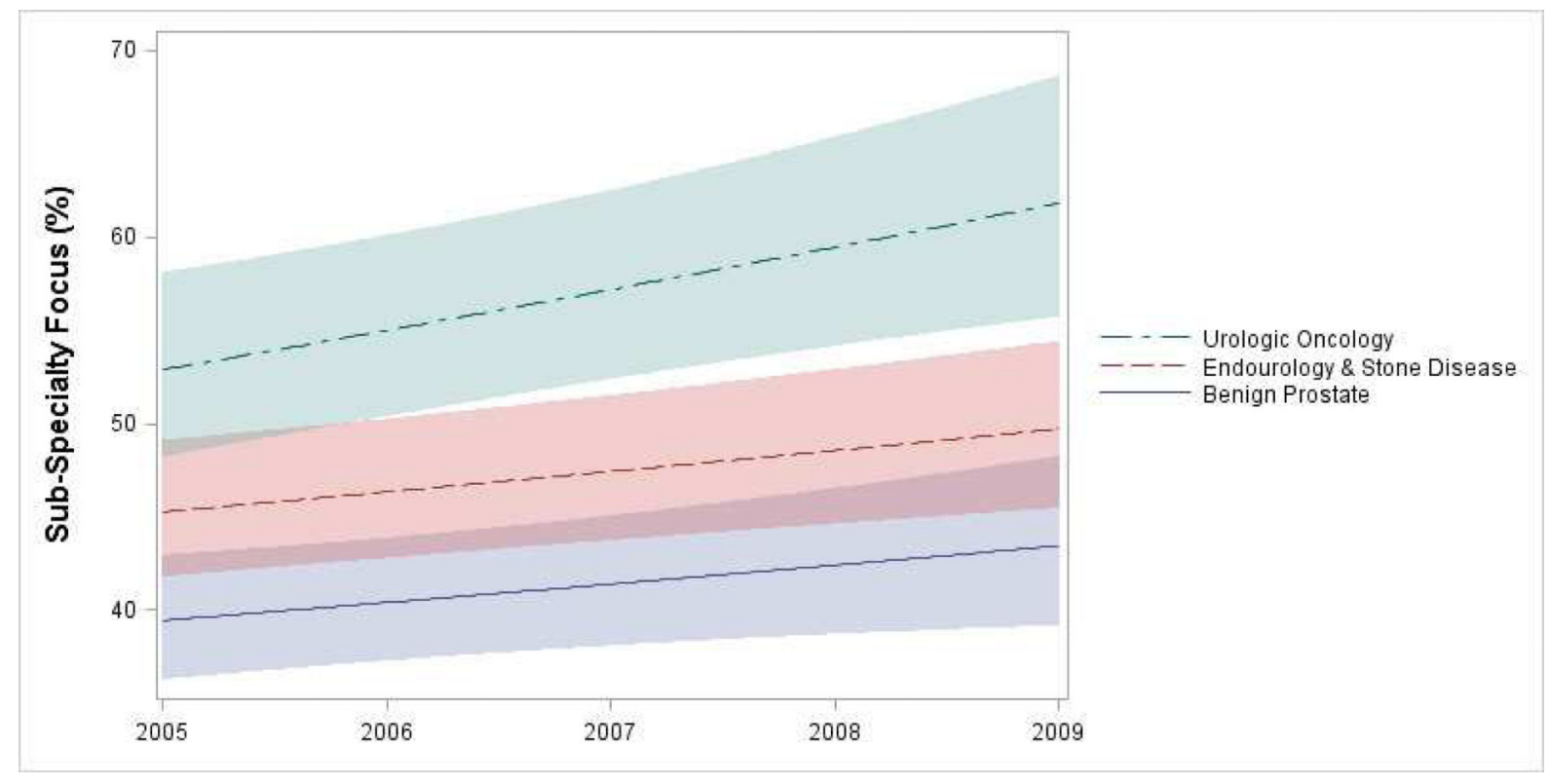

Figure 3. Longitudinal trends in subspecialty-focus according to clinical domain Trends in subspecialty-focus for three clinical domains, with $95 \%$ confidence intervals (shaded areas). Subspecialty focus increased significantly over time for urologists assigned to Urologic Oncology, Endourology \& Stone Disease, and Benign Prostate domains ( $p<0.001$ for each comparison). The rate of change in subspecialty-focus was statistically similar for urologists in all three domains. 


\section{Table 1}

Urological surgeons' predominant clinical domain by year.

\begin{tabular}{lccccc}
\hline & \multicolumn{5}{c}{ Year } \\
\cline { 2 - 6 } & $\mathbf{2 0 0 5}$ & $\mathbf{2 0 0 6}$ & $\mathbf{2 0 0 7}$ & $\mathbf{2 0 0 8}$ & $\mathbf{2 0 0 9}$ \\
\hline Individual surgeons, $n$ & 541 & 662 & 663 & 687 & 665 \\
Predominant clinical domain of procedures, \% & & & & & \\
Endourology \& Stone Disease & 44 & 47 & 42 & 47 & 49 \\
Urologic Oncology & 29 & 30 & 37 & 35 & 33 \\
Benign Prostate & 18 & 16 & 15 & 12 & 13 \\
Urogenital Reconstruction & 4 & 4 & 3 & 3 & 3 \\
Incontinence & 4 & 2 & 2 & 2 & 1 \\
Renal Transplant & 0 & 0 & 0 & 0 & 1 \\
Other & 1 & 1 & 1 & 1 & 0 \\
\hline
\end{tabular}

